\title{
EVOLUTION OF PARTICLE SEPARATION IN SLOWLY DECORRELATING VELOCITY FIELDS*
}

\author{
TOMASZ KOMOROWSKI ${ }^{\dagger}$, ALEXEI NOVIKOV ${ }^{\ddagger}$, AND LENYA RYZHIK ${ }^{\S}$
}

\begin{abstract}
We consider the evolution of the separation distance between two particles advected by a random velocity field with slowly decaying temporal and spatial correlations in the weak coupling regime. It has been shown in [5] that the motion of a single particle converges to a fractional Brownian motion on a time scale $\delta^{-\gamma}$ with some $\gamma<2$, which is shorter than the classical diffusive time scale $\delta^{-2}$ (see [9]). In the present paper we prove that unlike the single particle position, the two-particle separation behaves diffusively, and evolves on the classical time scale $\delta^{-2}$, even when the random flow is slowly decorrelating in time and space. The results of this paper illustrate that the flows under consideration display both diffusive and superdiffusive transport on different time scales for various physical quantities.
\end{abstract}

Key words. Two-particle separation distance, random velocity field, weak coupling regime, diffusive time scale.

AMS subject classifications. 34F05, 60F05.

\section{Introduction}

Motion of a particle in a random velocity field. We consider the motion of a particle advected by a weakly random velocity field $V(t, x)$ :

$$
\dot{X}(t)=\delta V(t, X(t)), \quad X(0)=x \in \mathbb{R}^{d} .
$$

Here $\delta \ll 1$ is a small parameter measuring the strength of the flow. This problem has been intensively studied; see the comprehensive review [12] for extensive references. The basic result is that under appropriate mixing and time-stationarity assumptions on the field $V(t, x)$, the rescaled process $x_{\delta}(t)=X\left(t / \delta^{2}\right)$ converges as $\delta \rightarrow 0$ to a diffusion process with the diffusion matrix given by the Kubo-Taylor formula

$$
D_{p q}=\int_{0}^{\infty} \mathbb{E}\left[V_{p}(t, 0) V_{q}(0,0)+V_{q}(t, 0) V_{p}(0,0)\right] d t, \quad p, q=1, \ldots, d .
$$

Here $\mathbb{E}$ denotes the expectation with respect to the realizations of the field.

This result, obviously, can hold only if the temporal correlations of $V(t, x)$ decay sufficiently fast so that the entries of the matrix $\left[D_{p q}\right]$ is finite. The situation is quite different when they are infinite. One particular example of such velocity field was considered in [5] when $V(t, x)$ is an Ornstein-Uhlenbeck process given by

$$
V(t, x):=\int e^{i k \cdot x} \hat{V}(t, d k),
$$

with $\hat{V}(t, d k)$ a stationary, stochastic measure-valued process defined by

$$
\hat{V}(t, d k)=\sqrt{2} \int_{-\infty}^{t} e^{-|k|^{2 \beta}(t-s)}|k|^{\beta} \widehat{\mathcal{B}}(d s, d k) .
$$

*Received: March 2, 2011; accepted (in revised version): September 4, 2011. Communicated by Jack Xin.

${ }^{\dagger}$ Institute of Mathematics, UMCS, pl. Marii Curie-Skłodowskiej 1, 20-031, Lublin and IMPAN, ul. Śniadeckich 8, 00-956 Warsaw, Poland (komorow@hektor.umcs.lublin.pl).

${ }^{\ddagger}$ Department of Mathematics, Pennsylvania State University, State College PA 16802, USA (anovikov@math.psu.edu).

$\S$ Department of Mathematics, Stanford University, Stanford, CA 94305, USA (ryzhik@math. stanford.edu). 
Here $\widehat{\mathcal{B}}(d t, d k)$ is an $\mathbb{R}^{d}$-valued, space-time noise, that is, a Gaussian, distribution valued process satisfying $\widehat{\mathcal{B}}^{*}(d t, d k)=\widehat{\mathcal{B}}(d t,-d k)$ and

$$
\mathbb{E}\left[\widehat{\mathcal{B}}_{i}(d t, d k) \widehat{\mathcal{B}}_{j}^{*}\left(d t^{\prime}, d k^{\prime}\right)\right]=\hat{R}_{i j}(k) \delta\left(t-t^{\prime}\right) \delta\left(k-k^{\prime}\right) d t d t^{\prime} d k d k^{\prime}
$$

with $\beta \geq 0$ and the spatial power spectrum given by

$$
\hat{R}(k)=\frac{a(|k|)}{|k|^{2 \alpha+d-2}}\left(I-\frac{k \otimes k}{|k|^{2}}\right) .
$$

The function $a(\cdot)$ is non-negative, bounded, measurable, supported in $\left[0, K_{0}\right]$ for some $K_{0}>0$, and continuous at 0 with $a(0)>0$. In order to ensure that the spectrum is integrable at $k=0$ so that $V(t, x)$ is a vector valued, stationary random field, we assume that $\alpha<1$. Informally, $\hat{V}(t, d k)$ is a stationary solution of the stochastic differential equation

$$
d \hat{V}_{i}(t, d k)=-|k|^{2 \beta} \hat{V}_{i}(t, d k) d t+\sqrt{2}|k|^{\beta} \widehat{\mathcal{B}}_{i}(d t, d k), \quad i=1, \ldots, d .
$$

The covariance matrix of $V(t, x)$ is

$$
R_{i j}(t, x)=\mathbb{E}\left[V_{i}(t, x) V_{j}(0,0)\right]=\int e^{i k \cdot x} e^{-|k|^{2 \beta}|t|} \hat{R}_{i j}(k) d k, \quad i, j=1, \ldots, d .
$$

It is straightforward to verify that the diffusion matrix given by the Kubo-Taylor formula (1.2) is finite if and only if $\alpha+\beta<1$. It has been shown in [4] that then in fact the process $x_{\delta}(t)=X\left(t / \delta^{2}\right)$ converges in law to a Brownian motion with the diffusivity matrix given by (1.2).

On the other hand, it has been shown in [5] that in the opposite regime $\alpha+\beta>1$ (when $\beta>0, \alpha<1$ ) the result is as follows. Because of the slow decay of the temporal correlations of the velocity field, the process $X(t)$ becomes non-trivial on a shorter time scale $t \sim O\left(\delta^{-2 \gamma}\right)$ with $\gamma=\beta /(\alpha+2 \beta-1)<1$. Hence, one should consider the process $x_{\delta}(t)=X\left(t / \delta^{2 \gamma}\right)$, which, in the limit $\delta \rightarrow 0$, converges to a superdiffusive fractional Brownian motion $B_{H}(t)$, with the Hurst exponent

$$
H=\frac{1}{2}+\frac{\alpha+\beta-1}{2 \beta} \in(1 / 2,1)
$$

Similar (but less sharp) results have been obtained in $[10,11]$ for a particle in a Gaussian time-independent velocity field with a large mean and slowly decaying correlations.

Behavior of the two-particle separation. One may also consider the evolution of the separation between a pair of particles advected by such a random flow: let $X(t, x)$ and $X(t, x+z)$ be two trajectories of (1.1) starting at the points $x$ and $x+z$, respectively, and set $z_{\delta}(t)=X\left(t / \delta^{2}, x+z\right)-X\left(t / \delta^{2}, x\right)$. The processes $x_{\delta}(t)$ and $z_{\delta}(t)$ satisfy the system

$$
\begin{aligned}
& \dot{x}_{\delta}(t)=\frac{1}{\delta} V\left(\frac{t}{\delta^{2}}, x_{\delta}(t)\right), \quad x_{\delta}(0)=0 \\
& \dot{z}_{\delta}(t)=\frac{1}{\delta} W\left(\frac{t}{\delta^{2}}, x_{\delta}(t), z_{\delta}(t)\right), \quad z_{\delta}(0)=z
\end{aligned}
$$


where

$$
W(t, x, z):=V(t, x+z)-V(t, x) .
$$

When the velocity field $V(t, x)$ is as above (see (1.3)-(1.7)), the random field $W(t, x, z)$ is given by

$$
W(t, x, z)=\sqrt{2} \int_{-\infty}^{t} \int e^{-|k|^{2 \beta}(t-s)} e^{i k \cdot x}\left(e^{i k \cdot z}-1\right)|k|^{\beta} \widehat{\mathcal{B}}(d s, d k) .
$$

The two-particle separation was also considered in [9]. It was shown that for rapidly decorrelating (in time and space) fields the process $z_{\delta}(t)$ converges weakly, as $\delta \rightarrow 0$, to a diffusion process with the diffusion matrix

$$
c_{p q}(z):=\int_{0}^{\infty}\left[R_{p q}(t, 0)+R_{q p}(t, 0)-R_{p q}(t, z)-R_{q p}(t, z)\right] d t .
$$

When the two-point correlation function has the form (1.5)-(1.7), this matrix takes the form

$$
c_{p q}(z)=\int \frac{1-\cos (k \cdot z)}{|k|^{2 \beta}} \hat{R}_{p q}(k) d k, \quad z \in \mathbb{R}^{d}, p, q=1, \ldots, d .
$$

A direct calculation shows that $\left|c_{p q}(z)\right|<+\infty$ for $1<\alpha+\beta<2$, which is a larger range of the parameters $\alpha$ and $\beta$ than that for which the "one-particle" diffusion matrix $D_{p q}$ given by (1.2) remains finite. In particular, in the range $1<\alpha+\beta<2$ the matrix $D_{p q}$ is infinite while $c_{p q}$ is finite. In agreement with this calculation, it was observed in [5] that, even when $\alpha+\beta>1$, the process $Z(t)$ behaves trivially on the "fractional diffusion" time scale $t \sim O\left(\delta^{-2 \gamma}\right): Z\left(t / \delta^{2 \gamma}\right) \approx z$. That is, all individual particles starting at positions separated by distance $z \sim O(1)$ perform perfectly correlated fractional Brownian motions on this time scale, and thus move together as a group.

A natural question addressed in the present paper is to find the time scale on which the particle separation has a non-trivial limit. It turns out that in the range $1<\alpha+\beta<2$ the two particle separation (unlike the one-particle position) becomes non-trivial on the "diffusion" time scale $t \sim O\left(\delta^{-2}\right)$, that is, much larger than the time scale $t \sim O\left(\delta^{-2 \gamma}\right)$, when one particle position has a non-trivial limit. Our main result can be formulated as follows.

Theorem 1.1. Let $Q_{\delta}$ be the law of $\left\{z_{\delta}(t), t \geq 0\right\}$ on $C[0,+\infty)$. Suppose that $\alpha+\beta>1$ and $\alpha+2 \beta<2$. Then, the family of measures $\left\{Q_{\delta}, \delta>0\right\}$ converges weakly, as $\delta \rightarrow 0+$, to the law of a diffusion with the generator given by

$$
L f(z)=\sum_{p, q=1}^{d} c_{p q}(z) \partial_{p, q}^{2} f(z)
$$

for any function $f(z)$ that is twice continuously differentiable.

We should mention that we do not try to identify the behavior of the individual particles on the time scale $O\left(\delta^{-2}\right)$ but rather only of the particle separation. Theorem 1.1 shows that slowly decaying temporal and spatial correlations of the random flow bring about multiple temporal scales: while the one-particle quantities evolve on the shorter "anomalous" time scale $O\left(\delta^{-2 \gamma}\right)$, for some $\gamma \in(0,1)$, the two particle separation evolves on a longer "classical diffusive" time scale. Similar temporal separation 
was observed in one-dimensional wave propagation $[13,6]$ and wave propagation in higher dimensions $[2,7]$. We believe that this very interesting phenomenon is an important characteristic feature of media with long range correlations in a large class of problems.

A Gaussian field with the covariance matrix given by (1.7) is statistically isotropic, in the sense that for any matrix $g \in S O(d)$ the laws of the fields $\left\{V(t, g x),(t, x) \in \mathbb{R}^{1+d}\right\}$ and $\left\{g V(t, x),(t, x) \in \mathbb{R}^{1+d}\right\}$ are identical. This hypothesis simplifies our computations but does not seem to be crucial for the validity of our result. Namely, we conjecture that, at the cost of some additional complication of calculations one should be able to prove that if the covariance matrix of the field is given by

$$
R(t, x)=\int_{\mathbb{R}} e^{i k \cdot x} e^{-r(k) t} \hat{R}(k) d k,
$$

where $\operatorname{tr} \hat{R}(k) \sim|k|^{-(2 \alpha+d-2)}$ and $r(k) \sim|k|^{2 \beta}$ for $|k| \ll 1$, and $\alpha, \beta$ are as in the statement of Theorem 1.1, then the conclusion of the theorem is still correct. Note also that the presence of the factor $I-k \otimes k /|k|^{2}$ in formula (1.5) ensures that the realizations of the random field are almost surely divergence free. The role of incompressibility in our result does not seem to be crucial either. If it does not hold the limiting diffusion would pick up a drift term, which vanishes in our situation; see condition (3.39) below. However, it should be noted that incompressibility has played a role in establishing the limit for the single particle motion in [5].

\section{Proof of Theorem 1.1}

The cut-off process. The idea of the proof is quite similar to that in [9]: one introduces a regularized process $z_{\delta}^{(M)}(t)$ that "does not behave wildly" for a finite value of the cut-off parameter $M$. One first establishes convergence for the regularized process, and then uses the "uniformly nice in $M$ " properties of the limit to establish the convergence of the original, unregularized process $z_{\delta}(t)$.

More precisely, given $M>1$, we consider a cut-off function $\phi_{M}$ belonging to $C_{0}^{\infty}\left(\mathbb{R}^{d}\right)$ - the space of infinitely smooth, compactly supported functions - such that $\phi_{M}(z)=1$ when $|z| \leq M-1$, and $\phi_{M}(z)=0$ when $|z| \geq M$. Let $W^{(M)}(t, z, x):=$ $W(t, z, x) \phi_{M}(z)$ and let $\left(x_{\delta}(t), z_{\delta}^{(M)}(t)\right)$ be the solution of

$$
\begin{aligned}
\dot{x}_{\delta}(t) & =\frac{1}{\delta} V\left(\frac{t}{\delta^{2}}, x_{\delta}(t)\right), \\
\dot{z}_{\delta}^{(M)}(t) & =\frac{1}{\delta} W^{(M)}\left(\frac{t}{\delta^{2}}, x_{\delta}(t), z_{\delta}^{(M)}(t)\right), \\
x_{\delta}(0) & =0, \quad z_{\delta}^{(M)}(0)=z_{0} .
\end{aligned}
$$

The regularization ensures that $\left|z_{\delta}^{(M)}(t)\right| \leq M$ for all $t \geq 0$. The first step in the proof of Theorem 1.1 is to establish tightness for the regularized processes $z_{\delta}^{(M)}(t)$.

Theorem 2.1. Suppose that $M>1$ is fixed. Then the family of laws of $\left\{z_{\delta}^{(M)}(t), t \geq 0\right\}$ is tight on $C[0,+\infty)$ as $\delta \rightarrow 0+$.

With this result in hand, by an argument in step (vi) of the proof of Theorem 3 of [9], tightness of the laws $Q_{\delta}$ of the process $z_{\delta}(t)$ follows from the tightness of the laws $Q_{\delta, M}$ of $z_{\delta}^{(M)}(t)$. 
Next, we identify the possible limits $Q_{M}^{*}$ of the laws $Q_{\delta, M}$ of the processes $z_{\delta}^{(M)}$, as $\delta \rightarrow 0+$. For any path $\pi \in C[0,+\infty)$ and $t \geq 0$, let $\Pi_{t}(\pi):=\pi(t)$. Denote by $\mathcal{M}_{t}$ the natural filtration corresponding to the canonical process $\left\{\Pi_{t}, t \geq 0\right\}$, and by $C_{b}^{m}\left(\mathbb{R}^{d}\right)$ the space of functions possessing $m$ continuous and bounded derivatives. We introduce also the regularized coefficients

$$
c_{p q}^{(M)}(z):=\phi_{M}^{2}(z) c_{p q}(z),
$$

with $c_{p q}(z)$ given by (1.11), and the drift

$$
b_{p}^{(M)}(z):=\frac{1}{2} \sum_{q=1}^{d} \partial_{z_{q}}\left[\phi_{M}^{2}(z)\right] c_{p q}(z) .
$$

The drift $b^{(M)}$ appears only because of the regularization that violates the incompressibility constraint in the $z$-component.

Theorem 2.2. Suppose that $1<\alpha+\beta$ and $\alpha+2 \beta<2$. Then, for any $f \in C_{b}^{2}\left(\mathbb{R}^{d}\right)$, $M>1$, and $\Psi_{s}$ that is bounded and $\mathcal{M}_{s}$-measurable, we have

$$
\int\left\{\left[f\left(\Pi_{t}\right)-f\left(\Pi_{s}\right)-\int_{s}^{t} L_{M} f\left(\Pi_{\rho}\right) d \rho\right] \Psi_{s}\right\} Q_{M}^{*}(d \pi)=0,
$$

where

$$
L_{M} f(z)=\sum_{p, q=1}^{d} c_{p q}^{(M)}(z) \partial_{p, q}^{2} f(z)+\sum_{p=1}^{d} b_{p}^{(M)}(z) \partial_{p} f(z) .
$$

Thanks to $C^{2}$ smoothness of the coefficients of the operator $L_{M}$ we conclude that for each $M>1$ there exists a unique limiting measure $Q_{M}^{*}$; see [15], Corollary 8.1.7. Hence $Q_{M, \delta} \Rightarrow Q_{M}^{*}$ as $\delta \rightarrow 0+$.

Theorem 1.1 as a consequence of Theorems 2.1 and 2.2. To obtain the weak convergence of $Q_{\delta}$ as $\delta \rightarrow 0+$, we consider the stopping time

$$
\tau_{M}(\pi):=\inf [t \geq 0:|\pi(t)| \geq M-1] \text { for a given } \pi \in C[0,+\infty),
$$

with the usual convention $\tau_{M}=+\infty$ if the infimum is taken over an empty set. Observe that $Q_{\delta}$ coincides with $Q_{\delta, M}$ when restricted to $\mathcal{M}_{\tau_{M}}$ - the $\sigma$-algebra consisting of those sets $A$ that $A \cap\left[\tau_{M} \leq t\right] \in \mathcal{M}_{t}$ for all $t \geq 0$. Hence, also $Q_{M}^{*}$ and $Q_{M^{\prime}}^{*}$ coincide on that $\sigma$-algebra when $M \leq M^{\prime}$. The family $\left\{Q_{M}^{*}, M \geq 1\right\}$ is tight as $M \rightarrow+\infty$. Indeed, it suffices only to show that for any $T, \varepsilon>0$ there exists $M_{0}$ such that

$$
Q_{M}^{*}\left(\tau_{M_{0}} \leq T\right)<\varepsilon, \quad \forall M \geq M_{0} .
$$

This follows from elementary properties of diffusions; see e.g. (2.1), p. 88 of [15].

As an immediate consequence of Theorem 2.2 we conclude that any $Q_{*}$ that is a limiting measure of $\left\{Q_{M}^{*}, M \geq 1\right\}$, as $M \rightarrow+\infty$, must satisfy the martingale problem

$$
\int\left\{\left[f\left(\Pi_{t}\right)-f\left(\Pi_{s}\right)-\int_{s}^{t} L f\left(\Pi_{\varrho}\right) d \varrho\right] \Psi_{s}\right\} Q_{*}(d \pi)=0
$$

for any $f \in C_{b}^{2}\left(\mathbb{R}^{d}\right)$ and $\Psi_{s}$ that is bounded and $\mathcal{M}_{s}$-measurable. Here operator $L$ is given by (1.12). Thus $Q_{M}^{*} \Rightarrow Q_{*}$, as $M \rightarrow+\infty$, and Theorem 1.1 follows.

Therefore, the demonstration of Theorem 1.1 is reduced to the proof of Theorems 2.1 and 2.2 . 
A martingale estimate. Both Theorems 2.1 and 2.2 are consequences of the following estimate. We denote by $\|f\|_{R, m}$ the $C^{m}$-norm of the function $f$ over the ball centered at 0 of radius $R>0$ and by $\mathcal{F}_{t}, t \geq 0$ the natural filtration corresponding to $\{V(t, \cdot), t \geq 0\}$.

THEOREM 2.3. Under the assumptions of Theorem 2.2 for any $M>1$ there exist constants $C, \epsilon>0$ and $\gamma \in(1,2)$ such that

$$
\begin{aligned}
& \left|\mathbb{E}\left\{\left[f\left(z_{\delta}^{(M)}(u)-Y\right)-f\left(z_{\delta}^{(M)}(t)-Y\right)-\int_{t}^{u} L_{M} f\left(z_{\delta}^{(M)}(s)-Y\right) d s\right] \Psi\right\}\right| \\
\leq & C\|\Psi\|_{L^{2}}\|f\|_{4 M, 3} \delta^{\epsilon}(u-t), \quad \forall \delta \in(0,1],
\end{aligned}
$$

where $t \geq \delta^{\gamma}, u-t \geq \delta^{\gamma}, f \in C^{3}\left(\mathbb{R}^{d}\right)$ are arbitrary, $L_{M}$ is given by (2.5), and $\Psi, Y$ are $\mathcal{F}_{t / \delta^{2}}$-measurable random variables with $\|Y\|_{L^{\infty}} \leq 2 M$.

Proofs of Theorems 2.1 and 2.2. Theorem 2.2 is a direct consequence of Theorem 2.3 and the tightness claim made in Theorem 2.1. We turn to the proof of Theorem 2.1. Let $Z_{\delta}(t):=z_{\delta}^{(M)}\left(t_{i+1}\right)$ for $t \in\left[t_{i}, t_{i+1}\right)$, with $\Delta t_{i}=\delta^{\gamma}$, and $\gamma$ as in the statement of Theorem 2.3. We show in Corollary 3.7 below that for each $\rho>0$ and $T>0$, we have

$$
\lim _{\delta \rightarrow 0+} Q_{\delta, M}\left(\sup _{t \in[0, T]}\left|Z_{\delta}(t)-z_{\delta}^{(M)}(t)\right| \geq \rho\right)=0 .
$$

Therefore, tightness of $Q_{\delta, M}$ follows if we show tightness of the laws of $\left\{Z_{\delta}(t), t \geq 0\right\}$ over $D[0,+\infty)$, as $\delta \rightarrow 0+$.

Suppose that $u-t \geq \delta^{\gamma}$ and $\Psi$ is a random variable that is $\mathcal{F}_{t / \delta^{2}}$-measurable. Then, we have

$$
\left|\mathbb{E}\left\{\left|Z_{\delta}(u)-Z_{\delta}(t)\right|^{2 i} \Psi\right\}\right|=\left|\mathbb{E}\left\{\left|z_{\delta}^{(M)}\left(t_{l}\right)-z_{\delta}^{(M)}\left(t_{k}\right)\right|^{2 i} \Psi\right\}\right|,
$$

for some $l \geq k \geq 1$ such that $0<t_{l}-u<\delta^{\gamma}, 0<t_{k}-t<\delta^{\gamma}$. We use Theorem 2.3, with $f(x)=|x|^{2 i}, i=1, \ldots, 4$ and $Y=z_{\delta}^{(M)}\left(t_{k}\right)$. According to this result, there exist constants $C, C^{\prime}>0$, possibly depending on $M$, but independent of the random variable and $\delta>0$, such that the right hand side of (2.9) is less than or equal to

$$
C\left(t_{l}-t_{k}\right)\left(\mathbb{E} \Psi^{2}\right)^{1 / 2} \leq C^{\prime}(u-t)\left(\mathbb{E} \Psi^{2}\right)^{1 / 2}, \quad i=1, \ldots, 4
$$

for all $\Psi$ that are $\mathcal{F}_{t / \delta^{2}}$ measurable.

Using this estimate with $\Psi \equiv 1$, we get

$$
\left|\mathbb{E}\left\{\left|Z_{\delta}(u)-Z_{\delta}(t)\right|^{2 i}\right\}\right| \leq C(u-t), \quad i=1, \ldots, 4 .
$$

Next, let $\Psi:=\left|Z_{\delta}(t)-Z_{\delta}(s)\right|^{4}$ for $t>s$. As $\Psi=\left|z_{\delta}^{(M)}\left(t_{k}\right)-Z_{\delta}(s)\right|^{4}$, it is $\mathcal{F}_{t_{k} / \delta^{2-}}$ measurable. When $t-s>\delta^{\gamma}$ we can apply again Theorem 2.3 and obtain, using $(2.11)$,

$$
\begin{aligned}
& \left|\mathbb{E}\left\{\left|Z_{\delta}(u)-Z_{\delta}(t)\right|^{4}\left|Z_{\delta}(t)-Z_{\delta}(s)\right|^{4}\right\}\right|=\left|\mathbb{E}\left\{\left|z_{\delta}^{(M)}\left(t_{l}\right)-z_{\delta}^{(M)}\left(t_{k}\right)\right|^{4} \Psi\right\}\right| \\
\leq & C(u-t)\left\{\mathbb{E}\left|Z_{\delta}(t)-Z_{\delta}(s)\right|^{8}\right\}^{1 / 2} \leq C(u-t)(t-s)^{1 / 2} \leq C(u-s)^{3 / 2}
\end{aligned}
$$


If on the other hand $0<t-s<\delta^{\gamma}$, then from Theorem 2.1 and Corollary 3.4 below we get for some $1<\gamma^{\prime}<\gamma$

$$
\begin{aligned}
& \left|\mathbb{E}\left\{\left|Z_{\delta}(u)-Z_{\delta}(t)\right|^{4}\left|Z_{\delta}(t)-Z_{\delta}(s)\right|^{4}\right\}\right| \leq C(u-t)\left\{\mathbb{E}\left|Z_{\delta}(t)-Z_{\delta}(s)\right|^{8}\right\}^{1 / 2} \\
\leq & C(u-t) \delta^{4\left(\gamma^{\prime}-1\right)} \leq C(u-s)^{1+4\left(\gamma^{\prime}-1\right) / \gamma} .
\end{aligned}
$$

Finally, we consider the situation when $0<u-t<\delta^{\gamma}$. Then only the case $t-s>\delta^{\gamma}$ requires our attention, since otherwise $\left|Z_{\delta}(u)-Z_{\delta}(t)\right|^{4}\left|Z_{\delta}(t)-Z_{\delta}(s)\right|^{4}=0$. For a given $\epsilon_{1}>0$ we can write

$$
\mathbb{E}\left\{\left|Z_{\delta}(u)-Z_{\delta}(t)\right|^{4}\left|Z_{\delta}(t)-Z_{\delta}(s)\right|^{4}\right\} \leq C \delta^{4\left(\gamma^{\prime}-1\right)}\left\{\mathbb{E}\left|Z_{\delta}(t)-Z_{\delta}(s)\right|^{4\left(1+\epsilon_{1}\right)}\right\}^{1 /\left(1+\epsilon_{1}\right)} .
$$

We use Theorem 2.3, this time with $f(x)=|x|^{4\left(1+\epsilon_{1}\right)}, Y=Z_{\delta}(s)$, and $\Psi \equiv 1$. Then, the right hand side of (2.14) can be estimated by

$$
C^{\prime} \delta^{4\left(\gamma^{\prime}-1\right)}(t-s)^{1 /\left(1+\epsilon_{1}\right)} \leq C^{\prime}(u-s)^{4\left(\gamma^{\prime}-1\right) / \gamma+1 /\left(1+\epsilon_{1}\right)} .
$$

We choose $\epsilon_{1}>0$ so that $4\left(\gamma^{\prime}-1\right) / \gamma+1 /\left(1+\epsilon_{1}\right)>1$. The above considerations imply tightness of the laws of $\left\{Z_{\delta}(t), t \geq 0\right\}$ in $D[0,+\infty)$, by virtue of Theorem 15.6 of [3]. This concludes the proof of Theorem 2.1. The rest of the paper contains the proof of Theorem 2.3.

\section{Proof of Theorem 2.3}

3.1. Preliminaries on Gaussian random fields. We quote here an estimate of the supremum of the norm of a centered Gaussian field that will be of particular use for us in the sequel. We recall that for a centered, not necessarily stationary, Gaussian $d$-dimensional vector field $G(t, x ; \omega),(t, x) \in \mathbb{R}^{1+d}$, a d-ball centered at $\left(t_{0}, x_{0}\right)$ and of radius $\varrho$ is defined as a ball with the corresponding center and radius with respect to the pseudometric

$$
\mathrm{d}\left(t_{1}, x_{1} ; t_{2}, x_{2}\right)=\left[\mathbb{E}\left|G\left(t_{1}, x_{1}\right)-G\left(t_{2}, x_{2}\right)\right|^{2}\right]^{1 / 2} .
$$

Suppose that $\mathcal{R} \subset \mathbb{R}^{1+d}$. For a given $\epsilon>0$ we let $N(\epsilon)$ be the minimal number of d-balls with radius $\epsilon>0$ needed to cover $\mathcal{R}$. Let also

$$
\Sigma(G):=\sup _{(t, x) \in \mathcal{R}} \operatorname{tr} Q(t, x ; t, x),
$$

where $Q$ is the covariance matrix of $G$ :

$$
Q_{i j}(t, x, s, y)=\mathbb{E}\left(G_{i}(t, x) G_{j}(s, y)\right), \quad i, j=1, \ldots, d .
$$

We will use the following Borell-Fernique-Talagrand type of estimates of the tail probabilities for Gaussian fields, see, for instance, Theorem 5.4, p. 121 of [1].

Theorem 3.1. Suppose that

$$
N(\epsilon) \leq C_{1} \exp \left\{C_{1} \epsilon^{-2 /\left(1+C_{2}\right)}\right\}, \quad \epsilon \in(0,1]
$$

for some positive constants where $C_{1}, C_{2}>0$. Then there exist constants $C, \Lambda>0$ depending only on $C_{1}, C_{2}$ such that

$$
\mathbb{P}\left(\sup _{(t, x) \in \mathcal{R}}|G(t, x ; \omega)| \geq \lambda\right) \leq C \exp \left\{-\frac{\lambda^{2}}{8 \Sigma(G)}\right\}
$$


for all $\lambda \geq \Lambda$.

The aforementioned result is stated in [1] for all $\lambda>0$, but in order to guarantee that the constants appearing in estimate (3.2) depend only on parameters $C_{1}, C_{2}$ we need to assume that $\lambda$ is sufficiently large; see the proof of this theorem on p. 121 in [1]. As a simple corollary of the above result we obtain.

Corollary 3.2. Suppose that $V(t, x)$ is a stationary field given by (1.3). Then, for any fixed $\rho>0$ there exists a constant $C>0$ and a constant $\Lambda>0$ such that

$$
\mathbb{P}\left[\exists(t, x):|V(t, x)| \geq \Lambda\left(\log ^{1 / 2+\rho}(|t|+|x|+1)+n\right)\right] \leq C e^{-n^{2} / C},
$$

for all $n \geq 1$.

Proof. Consider a field

$$
G(t, x):=V(t, x)\left[\log ^{1 / 2+\rho}(|t|+|x|+1)+n\right]^{-1} .
$$

Choose an arbitrary $\epsilon>0$. The region $D:=\left[|x|+|t| \geq \exp \left\{\epsilon^{-2 /(1+2 \rho)}\right\}\right]$ can be covered by finitely many d-balls of radius $\epsilon$. Since the covariance matrix $R(t, x)$ is Lipschitz continuous, the metric $\mathrm{d}(\cdot ; \cdot)$ is Hölder continuous with respect to the Euclidean distance, with exponent $1 / 2$. Hence, the complement of $D$ can be covered by $C \exp \left\{2(d+1) \epsilon^{-2 /(1+2 \rho)}\right\}$ d-balls of radius $\epsilon$. Note also that $\Sigma(G) \leq C n^{-2}$. Hence, from Theorem 3.1 we conclude that there exists $\Lambda>0$ so that

$$
\mathbb{P}\left(\sup _{x \in \mathbb{R}^{d}}|G(x ; \omega)| \geq \Lambda\right) \leq C \exp \left\{-\frac{\Lambda^{2}}{8 \Sigma(G)}\right\} \leq C_{1} \exp \left\{-C_{2} n^{2}\right\}
$$

and (3.3) holds.

Corollary 3.3. For any $m>1$ there exists constants $\gamma_{1}, \gamma_{2}>0$ such that

$$
\mathbb{E}\left[\sup _{|t|+|x| \leq \delta^{-2}}|V(t, x)|^{m}\right] \leq \gamma_{1}\left(\log \delta^{-1}\right)^{m \gamma_{2}}
$$

for all $\delta \in(0,1 / 2)$.

Proof. Observe that for $\lambda>2\left[\log \left(1+\delta^{-1}\right)\right]^{1 / 2+\rho}$, the inequality $|V(t, x)| \geq 2 \Lambda \lambda$ implies

$$
|V(t, x)| \geq \Lambda\left\{[\log (1+|t|+|x|)]^{1 / 2+\rho}+\lambda\right\} .
$$

Thus, by virtue of Corollary 3.2, we obtain

$$
\mathbb{P}\left[\sup _{|t|+|x| \leq \delta^{-2}}|V(t, x)| \geq 2 \Lambda \lambda\right] \leq C_{1} e^{-C_{2} \lambda^{2}},
$$

and, as a result, for any $m>1$ we can write

$$
\mathbb{E}\left[\sup _{|t|+|x| \leq \delta^{-2}}|V(t, x)|^{m}\right] \leq C\left[\log \left(1+\delta^{-1}\right)\right]^{(1 / 2+\rho) m},
$$

which in turn implies (3.4). 
As an immediate consequence of Corollary 3.3 we get

Corollary 3.4. Suppose that $\alpha<1$. Then, for any $M>0$ and $m \geq 1$ there exist constants $C, \rho>0$ such that

$$
\left\{\mathbb{E}\left[\sup _{s \in[0, T]} \sup _{|z| \leq M,|x| \leq \delta^{-2}}\left|W\left(\frac{s}{\delta^{2}}, z, x\right)\right|^{m}\right]\right\}^{1 / m} \leq C\left[\log \left(1+\delta^{-1}\right)\right]^{(1 / 2+\rho)} .
$$

In our next result we bound the moments of the supremum of a $d$-dimensional Gaussian field $G(x)=\left(G_{1}(x), \ldots, G_{d}(x)\right), x \in \mathbb{R}^{N}$ over a given region using the bounds on its covariance. Suppose that the field is a.s. differentiable and

$$
\Sigma(G):=\sup _{x \in \mathbb{R}^{N}}\left[\mathbb{E}|G(x)|^{2}+\mathbb{E}\left|\nabla_{x} G(x)\right|^{2}\right] .
$$

For a given $k \in \mathbb{Z}^{N}$ denote $\square_{k}:=\left[x \in \mathbb{R}^{N}: \sup _{1 \leq j \leq N}\left|x_{j}-k_{j}\right| \leq 1\right]$. Define also $M(\mathcal{R})$ as the minimal number of boxes $\square_{k}$ needed to cover a given region $\mathcal{R}$. Assume furthermore that $\mathcal{R}_{\delta}, \delta \in(0,1]$ are (non-random) regions such that there exist $C_{0}, d_{0}>0$ such that

$$
M\left(\mathcal{R}_{\delta}\right) \leq C_{0} \delta^{-d_{0}}, \quad \delta \in(0,1] .
$$

Proposition 3.5. Under the above assumptions for any $m \geq 1$ and $\mu>0$ there exists a constant $C>0$, depending only on $d, N, C_{0}, d_{0}, m, \mu$ such that

$$
\mathbb{E}\left\{\sup _{x \in \mathcal{R}_{\delta}}|G(x)|^{m}\right\} \leq C \Sigma^{m / 2}(G) \delta^{-\mu}, \quad \forall \delta \in(0,1] .
$$

Proof. Suppose that $p>d$. It follows from the Sobolev embedding theorem that there exists a deterministic constant depending only on $N$ and $d$ such that

$$
\sup _{x \in \square_{k}}|G(x)| \leq C\left\{\int_{\square_{k}}\left(|G(x)|^{p}+\left|\nabla_{x} G(x)\right|^{p}\right) d x\right\}^{1 / p}, \quad \forall k \in \mathbb{Z}^{N} .
$$

This, in particular, implies (using Gaussianity of the field) that for any given integer $n \geq 1$ we have

$$
\mathbb{E}\left[\sup _{x \in \square_{k}}|G(x)|^{n m}\right] \leq C_{n} \Sigma^{m n / 2}(G)
$$

for some constant $C_{n}$, depending on $n$. Observe that

$$
\sup _{x \in \mathcal{R}_{\delta}}|G(x)|^{m} \leq\left\{\sum \sup _{x \in \square_{k}}|G(x)|^{n m}\right\}^{1 / n},
$$

where the summation extends over the smallest set of $k$-s for which $\mathcal{R}_{\delta} \subset \bigcup_{k} \square_{k}$. Jensen inequality implies that

$$
\mathbb{E}\left\{\sup _{x \in \mathcal{R}_{\delta}}|G(x)|^{m}\right\} \leq\left\{\sum \mathbb{E}\left[\sup _{x \in \square_{k}}|G(x)|^{n m}\right]\right\}^{1 / n} .
$$

Using (3.6) and (3.8) we obtain

$$
\mathbb{E}\left\{\sup _{x \in \mathcal{R}_{\delta}}|G(x)|^{m}\right\} \leq C\left\{\delta^{-2 d_{0}} \Sigma^{m n / 2}(G)\right\}^{1 / n}=C \delta^{-2 d_{0} / n} \Sigma^{m / 2}(G) \leq C \delta^{-\mu} \Sigma^{m / 2}(G),
$$

upon a choice of a sufficiently large $n$. 


\subsection{A priori bounds on trajectories.}

Proposition 3.6. Suppose that $\left(x_{\delta}(t), z_{\delta}(t)\right)$ are given by (1.8). Then for any fixed $T>0$ there exist constants $C_{1}, C_{2}>0$ such that

$$
\mathbb{P}\left[\sup _{t \in[0, T]}\left(\left|x_{\delta}(t)\right|+\left|z_{\delta}(t)\right|\right) \geq \delta^{-2}\right] \leq C_{1} e^{-C_{2} / \delta^{2}}, \delta \in(0,1] .
$$

Proof. We prove that

$$
\mathbb{P}\left[\sup _{t \in[0, T]}\left|x_{\delta}(t)\right| \geq C \delta^{-2}\right] \leq C_{1} e^{-C_{2} / \delta^{2}} .
$$

An analogous estimate then holds for $x_{\delta}(t)+z_{\delta}(t)$, hence the estimate for $z_{\delta}(t)$ would follow. Using (3.3) with $n=\delta^{-1}$, we conclude that

$$
\left|x_{\delta}(t)\right| \leq C \delta^{-1}\left(\int_{0}^{t} \log ^{1 / 2+\rho}\left(T \delta^{-2}+\left|x_{\delta}(s)\right|\right) d s+\delta^{-1}\right), \quad \forall t \in[0, T]
$$

outside an event $A(\delta)$ such that $\mathbb{P}(A(\delta)) \leq C_{1} e^{-C_{2} / \delta^{2}}$. But then (still, outside the event $A(\delta)$ )

$$
\log ^{1 / 2+\rho}\left(T / \delta^{2}+\left|x_{\delta}(s)\right|\right) \leq C_{3}\left(\left|x_{\delta}(t)\right|+1\right)+\frac{C_{3}}{\delta},
$$

so

$$
\left|x_{\delta}(t)\right| \leq \frac{C_{4}}{\delta}\left(\int_{0}^{t}\left|x_{\delta}(s)\right| d s+\delta^{-1}\right),
$$

which in turn implies that, by Gronwall's inequality,

$$
\left|x_{\delta}(t)\right| \leq C_{5} e^{C_{4}(t+1) \delta^{-1}} .
$$

Using (3.13) in (3.11) we obtain

$$
\left|x_{\delta}(t)\right| \leq C_{6} \delta^{-1}\left(\int_{0}^{t} \delta^{-1 / 2-\rho} d s+\delta^{-1}\right) .
$$

We use (3.11) with (3.14) to obtain

$$
\left|x_{\delta}(t)\right| \leq C_{7}\left(\delta^{-1} \int_{0}^{t} \log ^{-1 / 2-\rho} \delta^{-1} d s+\delta^{-1}\right) \leq C_{8} \delta^{-2}
$$

outside $A(\delta)$. Thus we obtain (3.10). Finally, (3.9) follows once we adjust the constants $C_{1}$ and $C_{2}$ in the right-hand side of (3.9) appropriately.

Let $T \geq 0$ be fixed, and define the event

$$
A_{\delta, T}:=\left[\sup _{s \in[0, T]}\left|x_{\delta}(s)\right| \geq \delta^{-2}\right] .
$$

Proposition 3.6 says, in particular, that

$$
\mathbb{P}\left[A_{\delta, T}\right] \leq C_{1} e^{-C_{2} \delta^{-2}}, \quad \forall \delta \in(0,1],
$$


with the constants $C_{1}$ and $C_{2}$ that may depend on $T$.

We also conclude the following.

Corollary 3.7. For each $\gamma>1$ and $T, M, \rho>0$ fixed we have

$$
\lim _{\delta \rightarrow 0+} \mathbb{P}\left(\sup _{s, t \in[0, T],|s-t| \leq \delta^{\gamma}}\left|z_{\delta}^{(M)}(t)-z_{\delta}^{(M)}(s)\right| \geq \rho\right)=0 .
$$

Proof. The probability under the limit can be estimated by

$$
\mathbb{P}\left(A_{\delta, T}\right)+\mathbb{P}\left(\sup _{s \in[0, T]} \sup _{|z| \leq M,|x| \leq \delta^{-2}}\left|W\left(\frac{s}{\delta^{2}}, z, x\right)\right| \geq \delta^{1-\gamma} \rho\right),
$$

and the conclusion follows from (3.16), Corollary 3.4, and an application of the Chebyshev inequality.

3.3. The proof of the martingale estimate. We now turn to the proof of the martingale estimate (2.8). We divide the interval $[t, u]$ into subintervals $\left[t_{i}, t_{i+1}\right]$, where $t_{i}:=t+i \Delta t$ and $\Delta t:=\delta^{\gamma}$, with $1<\gamma<2$. The last interval $\left[t_{K-1}, t_{K}\right]$ could be of size less than or equal to $\delta^{\gamma}$. To abbreviate the notation we shall also assume that $Y=0$, as its presence does not influence the argument. We write then

$$
\begin{aligned}
& \mathbb{E}\left\{\left[f\left(z_{\delta}^{(M)}(u)\right)-f\left(z_{\delta}^{(M)}(t)\right)\right] \Psi\right\}=\sum_{i} \mathbb{E}\left\{\left[f\left(z_{\delta}^{(M)}\left(t_{i+1}\right)\right)-f\left(z_{\delta}^{(M)}\left(t_{i}\right)\right)\right] \Psi\right\} \\
= & \sum_{i} \mathbb{E}\left\{\left[f\left(z_{\delta}^{(M)}\left(t_{i+1}\right)\right)-f\left(z_{\delta}^{(M)}\left(t_{i}\right)\right)\right] \Psi, A_{\delta, t_{i-1}}\right\} \\
& +\sum_{i} \mathbb{E}\left\{\left[f\left(z_{\delta}^{(M)}\left(t_{i+1}\right)\right)-f\left(z_{\delta}^{(M)}\left(t_{i}\right)\right)\right] \Psi, A_{\delta, t_{i-1}}^{c}\right\} .
\end{aligned}
$$

The summation extends from $i=0$ up to $K-1$. Observe also that since $t \geq \delta^{\gamma}$ we have $t_{-1} \geq 0$.

Denote the terms on the utmost right hand side of (3.18) by $K_{1}$ and $K_{2}$. It is easy to observe, using (3.16), that

$$
\left|K_{1}\right| \leq C\|\Psi\|_{L^{2}}\|f\|_{4 M, 0} \delta^{\epsilon+\gamma} \leq C\|\Psi\|_{L^{2}}\|f\|_{4 M, 0} \delta^{\epsilon}(u-t),
$$

for some $C, \epsilon>0$, as $u-t>\delta^{\gamma}$. In the rest of this proof we shall estimate $K_{2}$. Note that

$$
\begin{aligned}
& f\left(z_{\delta}^{(M)}\left(t_{i+1}\right)\right)-f\left(z_{\delta}^{(M)}\left(t_{i}\right)\right)=\frac{1}{\delta} \sum_{p=1}^{d} \int_{t_{i}}^{t_{i+1}} \partial_{p} f\left(z_{\delta}^{(M)}(s)\right) W_{p}^{(M)}\left(\frac{s}{\delta^{2}}, z_{\delta}^{(M)}(s), x_{\delta}(s)\right) d s \\
= & \frac{1}{\delta} \sum_{p=1}^{d} \int_{t_{i}}^{t_{i+1}} \partial_{p} f\left(z_{\delta}^{(M)}\left(t_{i-1}\right)\right) W_{p}^{(M)}\left(\frac{s}{\delta^{2}}, z_{\delta}^{(M)}\left(t_{i-1}\right), x_{\delta}\left(t_{i-1}\right)\right) d s \\
& +\frac{1}{\delta} \sum_{p=1}^{d} \int_{t_{i}}^{t_{i+1}}\left\{\int_{t_{i-1}}^{s} \frac{d}{d \rho}\left[\partial_{p} f\left(z_{\delta}^{(M)}(\rho)\right) W_{p}^{(M)}\left(\frac{s}{\delta^{2}}, z_{\delta}^{(M)}(\rho), x_{\delta}(\rho)\right)\right] d \rho\right\} d s
\end{aligned}
$$

We substitute this expression into (3.18) and denote the respective terms that arise from $K_{2}$ by $J_{1}$ and $J_{2}$. The reason that the decomposition (3.19) is helpful is that there is a "relatively large" time gap between the time $t_{i-1}$, and all times $s \in\left[t_{i}, t_{i+1}\right]$. This will allow us to use the mixing in time properties of the field $W(t, z, x)$ (which are slightly better than those of $V(t, x)$ ) to establish "near independence" of what happens at time $s$ and what happened before time $t_{i-1}$. 
The estimate of $J_{1}$. We can write

$$
J_{1}=\sum_{i} J_{1}^{(i)}
$$

with

$$
\begin{aligned}
J_{1}^{(i)} & =\frac{1}{\delta} \mathbb{E}\left\{\left[\sum_{p=1}^{d} \int_{t_{i}}^{t_{i+1}} \partial_{p} f\left(z_{\delta}^{(M)}\left(t_{i-1}\right)\right) W_{p}^{(M)}\left(\frac{s}{\delta^{2}}, z_{\delta}^{(M)}\left(t_{i-1}\right), x_{\delta}\left(t_{i-1}\right)\right) d s\right] \Psi, A_{\delta, t_{i-1}}^{c}\right\} \\
& =\frac{1}{\delta} \sum_{p=1}^{d} \int_{t_{i}}^{t_{i+1}} \mathbb{E}\left\{\partial_{p} f\left(z_{\delta}\left(t_{i-1}\right)\right) \Psi \bar{W}_{p}^{(M)}\left(\frac{s}{\delta^{2}}, \frac{t_{i-1}}{\delta^{2}}, z_{\delta}\left(t_{i-1}\right), x_{\delta}\left(t_{i-1}\right)\right), A_{\delta, t_{i-1}}^{c}\right\} d s .
\end{aligned}
$$

Here we have denoted

$$
\bar{W}^{(M)}(s, \tau, z, x):=\mathbb{E}\left\{W^{(M)}(s, z, x) \mid \mathcal{F}_{\tau}\right\}=\phi_{M}(z) \bar{W}(s, \tau, z, x)
$$

for any $s \geq \tau$, where

$$
\bar{W}(s, \tau, z, x):=\mathbb{E}\left\{W(s, z, x) \mid \mathcal{F}_{\tau}\right\} .
$$

Rewriting the formula for $\bar{W}(s, \tau, z, x)$ using the spectral decomposition (1.3) and (1.4) we get

$$
\begin{aligned}
\bar{W}(s, \tau, z, x) & =\mathbb{E}\left\{\int_{\mathbb{R}^{d}} e^{i k \cdot x}\left(e^{i k \cdot z}-1\right) \hat{V}(s, d k) \mid \mathcal{F}_{\tau}\right\} \\
& =\sqrt{2} \int_{-\infty}^{\tau} \int_{\mathbb{R}^{d}} e^{-|k|^{2 \beta}(s-\rho)}|k|^{\beta} e^{i k \cdot x}\left(e^{i k \cdot z}-1\right) \hat{\mathcal{B}}(d \rho, d k) .
\end{aligned}
$$

The next lemma gives an estimate of $J_{1}$ using the aforementioned gap between the time $t_{i-1}$ and times $s \in\left[t_{i}, t_{i+1}\right]$, as well as the sufficient mixing properties of the field $W(t, x, z)$.

Lemma 3.8. Suppose that $\alpha+2 \beta<2$ and $\ell=\left(\ell_{1}, \ldots, \ell_{d}\right)$ is a multi-index of nonnegative integers. Then, there exists $\epsilon>0$ so that

$$
\sup _{i} \sup _{s \in\left[t_{i}, t_{i+1}\right]}\left\{\mathbb{E}\left[\sup _{|z| \leq M,|x| \leq \delta^{-2}}\left|\partial_{z}^{\ell} \bar{W}_{p}\left(\frac{s}{\delta^{2}}, \frac{t_{i-1}}{\delta^{2}}, z, x\right)\right|^{2}\right]\right\}^{1 / 2} \leq C \delta^{1+\epsilon}, \quad \delta \in(0,1] .
$$

Proof. We only consider $\ell=0$. The case of other multi-indices can be treated similarly; indeed, the main difficulty here is that the correlation function of the random field $W$ decays rather slowly. However, each differentiation in $z$ improves the decay of the correlation function in space; hence, $\ell=0$ is actually the most difficult case.

Thanks to the time stationarity of the field $V(t, x)$ it suffices only to prove that

$$
\sup _{s \in\left[\delta^{\gamma-2}, 2 \delta^{\gamma-2}\right]}\left\{\mathbb{E}\left[\sup _{|z| \leq M,|x| \leq \delta^{-2}}|\bar{W}(s, 0, z, x)|^{2}\right]\right\}^{1 / 2} \leq C \delta^{1+\epsilon} .
$$


Since supp $a(\cdot) \subset\left[0, K_{0}\right]$ we have, for any $p=1, \ldots, d$,

$$
\begin{aligned}
& \mathbb{E}\left\{\bar{W}_{p}^{2}(s, 0, z, 0)\right\} \leq C\left[\phi_{M}(z)\right]^{2} \int_{-\infty}^{0} \int_{\mathbb{R}^{d}} e^{-2|k|^{2 \beta}(s-\rho)}|k|^{2 \beta} \operatorname{tr} \hat{R}(k)\left|e^{i k \cdot z}-1\right|^{2} d \rho d k(3.24) \\
\leq & C 1_{[0, M]}(|z|) \int_{|k| \leq K_{0}} e^{-2|k|^{2 \beta} s}\left|e^{i k \cdot z}-1\right|^{2} \frac{d k}{|k|^{2 \alpha+d-2}} \leq C M^{2} \int_{0}^{K_{0}} e^{-2|k|^{2 \beta} \delta^{\gamma-2}} \frac{d k}{k^{2 \alpha-3}} .
\end{aligned}
$$

Making the substitution $m=k \delta^{\frac{\gamma-2}{2 \beta}}$, where $1<\gamma<2$, we get that the last integral equals

$$
\delta^{(2-\alpha)(2-\gamma) / \beta} \int_{0}^{K_{0} \delta^{(\gamma-2) /(2 \beta)}} \frac{e^{-2 m^{2 \beta}} d m}{m^{2 \alpha-3}} \leq \delta^{(2-\alpha)(2-\gamma) / \beta} \int_{0}^{\infty} \frac{e^{-2 m^{2 \beta}} d m}{m^{2 \alpha-3}} .
$$

Assuming that $(2-\alpha) / \beta>2$, we can select $\gamma$ sufficiently close to 1 to ensure that the right hand side can be estimated by $C \delta^{2+2 \epsilon}$ for some $\epsilon>0$, as $\delta \rightarrow 0+$. This requirement amounts to $\alpha+2 \beta<2$. The conclusion of the lemma is a direct consequence of Proposition 3.5.

We have shown therefore the following.

Corollary 3.9. Under the assumptions of Theorem 2.2 there exist constants $C, \epsilon>0$ and $\gamma \in(1,2)$ such that for $u, t \in[0, T]$,

$$
\left|J_{1}\right| \leq C \delta^{\epsilon}\|\Psi\|_{L^{2}}\|f\|_{4 M, 1}(u-t), \quad \forall \delta \in(0,1], u-t \geq \delta^{\gamma} .
$$

The limit of $J_{2}$. As Corollary 3.9 shows, our task is reduced to finding the limit of the term $J_{2}$. We recall, for the convenience of the reader that

$$
\begin{aligned}
J_{2}=\sum_{i} \mathbb{E}\{ & {\left[\frac{1}{\delta} \sum_{p=1}^{d} \int_{t_{i}}^{t_{i+1}} \int_{t_{i-1}}^{s}\right.} \\
& \left.\left.\frac{d}{d \rho}\left[\partial_{p} f\left(z_{\delta}^{(M)}(\rho)\right) W_{p}^{(M)}\left(\frac{s}{\delta^{2}}, z_{\delta}^{(M)}(\rho), x_{\delta}(\rho)\right)\right] d \rho d s\right] \Psi, A_{\delta, t_{i-1}}^{c}\right\}
\end{aligned}
$$

We introduce, for $s>\rho>\tau$,

$$
\begin{aligned}
& W_{p q}^{(M)}(s, \rho, z, x):=W_{p}^{(M)}(s, z, x) W_{q}^{(M)}(\rho, z, x), \\
& \widehat{W}_{p q}^{(M)}(s, \rho, \tau, z, x)=\mathbb{E}\left\{W_{p q}^{(M)}(s, \rho, z, x) \mid \mathcal{F}_{\tau}\right\}, \\
& \bar{W}_{p q}^{(M)}(s, z):=\mathbb{E} W_{p q}^{(M)}(s, 0, z, 0) \\
& \widetilde{W}_{p q}^{(M)}(s, \rho, \tau, z, x):=\widehat{W}_{p q}^{(M)}(s, \rho, \tau, z, x)-\bar{W}_{p q}^{(M)}(s-\rho, z) .
\end{aligned}
$$

We also introduce the corresponding notions without the cut-off $\phi_{M}(z)$, with the superscript $M$ dropped, and also

$$
\widehat{W}_{p q}^{(M)}(s, \rho, z, x)=\widehat{W}_{p q}^{(M)}(s, \rho, \rho, z, x) .
$$

We further Taylor-expand the term in $J_{2}$ corresponding to a fixed $i$ as

$$
\frac{1}{\delta} \sum_{p=1}^{d} \int_{t_{i}}^{t_{i+1}} \int_{t_{i-1}}^{s} \frac{d}{d \rho}\left[\partial_{p} f\left(z_{\delta}^{(M)}(\rho)\right) W_{p}^{(M)}\left(\frac{s}{\delta^{2}}, z_{\delta}^{(M)}(\rho), x_{\delta}(\rho)\right)\right] d \rho d s
$$




$$
\begin{aligned}
= & \frac{1}{\delta^{2}} \sum_{p, q=1}^{d} \int_{t_{i}}^{t_{i+1}} \int_{t_{i-1}}^{s} \partial_{p q}^{2} f\left(z_{\delta}^{(M)}(\rho)\right) W_{p q}^{(M)}\left(\frac{s}{\delta^{2}}, \frac{\rho}{\delta^{2}}, z_{\delta}^{(M)}(\rho), x_{\delta}(\rho)\right) d \rho d s \\
& +\frac{1}{\delta^{2}} \sum_{p, q=1}^{d} \int_{t_{i}}^{t_{i+1}} \int_{t_{i-1}}^{s} \partial_{p} f\left(z_{\delta}^{(M)}(\rho)\right) \partial_{z_{q}} W_{p}^{(M)}\left(\frac{s}{\delta^{2}}, z_{\delta}^{(M)}(\rho), x_{\delta}(\rho)\right) \\
& W_{q}^{(M)}\left(\frac{\rho}{\delta^{2}}, z_{\delta}^{(M)}(\rho), x_{\delta}(\rho)\right) d \rho d s \\
& +\frac{1}{\delta^{2}} \sum_{p, q=1}^{d} \int_{t_{i}}^{t_{i+1}} \int_{t_{i-1}}^{s} \partial_{p} f\left(z_{\delta}^{(M)}(\rho)\right) \partial_{x_{q}} W_{p}^{(M)}\left(\frac{s}{\delta^{2}}, z_{\delta}^{(M)}(\rho), x_{\delta}(\rho)\right) V_{q}\left(\frac{\rho}{\delta^{2}}, x_{\delta}(\rho)\right) d \rho d s .
\end{aligned}
$$

Denote the respective terms that arising from $J_{2}$ by $J_{21}, J_{22}, J_{23}$ respectively. We will show that these terms have the following limits.

Proposition 3.10. There exists constants $C, \epsilon>0$ such that

$$
\begin{aligned}
& \left|J_{21}-\sum_{i} \sum_{p, q=1}^{d} \int_{t_{i}}^{t_{i+1}} \mathbb{E}\left\{\partial_{p q}^{2} f\left(z_{\delta}^{(M)}\left(t_{i-1}\right)\right) c_{p q}^{(M)}\left(z_{\delta}^{(M)}\left(t_{i-1}\right)\right) \Psi, A_{\delta, t_{i-1}}^{c}\right\} d s\right| \\
\leq & C \delta^{\epsilon}(u-t)\|f\|_{4 M, 3}\|\Psi\|_{L^{2}}, \quad \forall \delta \in(0,1], u-t \geq \delta^{\gamma} .
\end{aligned}
$$

Proposition 3.11. There exists constants $C, \epsilon>0$ such that

$$
\begin{aligned}
& \quad\left|J_{22}-\frac{1}{2} \mathbb{E}\left[\Psi \sum_{q=1}^{d} \int_{t}^{u}\left(c_{p q} \partial_{z_{q}} \phi_{M}^{2}\right)\left(z_{\delta}^{(M)}(s)\right) \partial_{p} f\left(z_{\delta}^{(M)}(s)\right) d s\right]\right| \\
& \leq C \delta^{\epsilon}(u-t)\|f\|_{4 M, 1}\|\Psi\|_{L^{2}}
\end{aligned}
$$

and

$$
\left|J_{23}\right| \leq C \delta^{\epsilon}(u-t)\|f\|_{4 M, 1}\|\Psi\|_{L^{2}}, \quad \forall \delta \in(0,1], u-t \geq \delta^{\gamma} .
$$

This will complete the proof of Theorem 2.3 as the non-trivial terms in (3.28) and (3.29) combine to produce the operator $L_{M}$; see formulas (2.2), (2.3), and (2.5).

The proof of Proposition 3.10. We have

$$
\begin{aligned}
J_{21}= & \frac{1}{\delta^{2}} \sum_{p, q=1}^{d} \sum_{i} \int_{t_{i}}^{t_{i+1}} \int_{t_{i-1}}^{s} \\
& \mathbb{E}\left\{\partial_{p q}^{2} f\left(z_{\delta}^{(M)}(\rho)\right) W_{p q}^{(M)}\left(\frac{s}{\delta^{2}}, \frac{\rho}{\delta^{2}}, z_{\delta}^{(M)}(\rho), x_{\delta}(\rho)\right) \Psi, A_{\delta, t_{i-1}}^{c}\right\} d s d \rho .
\end{aligned}
$$

We can further write

$$
J_{21}=A_{1}+A_{2}+A_{3}
$$

where $A_{j}=\sum_{i} A_{j}^{(i)}, j=1,2,3$, with

$$
\begin{array}{r}
A_{1}^{(i)}:=\frac{1}{\delta^{2}} \sum_{p, q=1}^{d} \int_{t_{i}}^{t_{i+1}} d s \int_{t_{i-1}}^{s} d \rho \int_{t_{i-1}}^{\rho} d \tau \mathbb{E}\left\{\frac { d } { d \tau } \left\{\partial_{p q}^{2} f\left(z_{\delta}^{(M)}(\tau)\right)\right.\right. \\
\left.\left.\times W_{p q}^{(M)}\left(\frac{s}{\delta^{2}}, \frac{\rho}{\delta^{2}}, z_{\delta}^{(M)}(\tau), x_{\delta}(\tau)\right)\right\} \Psi, A_{\delta, t_{i-1}}^{c}\right\}
\end{array}
$$




$$
\begin{aligned}
A_{2}^{(i)}:=\frac{1}{\delta^{2}} \sum_{p, q=1}^{d} \int_{t_{i}}^{t_{i+1}} d s \int_{t_{i-1}}^{s} d \rho \mathbb{E}\left\{\partial_{p q}^{2} f\left(z_{\delta}^{(M)}\left(t_{i-1}\right)\right)\right. \\
\left.\times \widetilde{W}_{p q}^{(M)}\left(\frac{s}{\delta^{2}}, \frac{\rho}{\delta^{2}}, \frac{t_{i-1}}{\delta^{2}}, z_{\delta}^{(M)}\left(t_{i-1}\right), x_{\delta}\left(t_{i-1}\right)\right) \Psi, A_{\delta, t_{i-1}}^{c}\right\} \\
A_{3}^{(i)}:=\frac{1}{\delta^{2}} \sum_{p, q=1}^{d} \int_{t_{i}}^{t_{i+1}} d s \int_{t_{i-1}}^{s} d \rho \mathbb{E}\left\{\partial_{p q}^{2} f\left(z_{\delta}^{(M)}\left(t_{i-1}\right)\right) \bar{W}_{p q}^{(M)}\left(\frac{s-\rho}{\delta^{2}}, z_{\delta}^{(M)}\left(t_{i-1}\right)\right) \Psi, A_{\delta, t_{i-1}}^{c}\right\} .
\end{aligned}
$$

We will see that the term $A_{1}$ is small since each $A_{1}^{(i)}$ involves three integrals over intervals of length smaller than $\delta^{\gamma}$, with $\gamma>1$. This produces a term of the order $\delta^{3 \gamma-2}$, which after summation over $i$ will still be of the order $\delta^{\epsilon}$ with $\epsilon>0$. The terms $A_{2}^{(i)}$ are small because of the time-mixing properties of the filed $W(t, x, z)$ : there is either a "large" gap between $s$ and $\rho$ or between $\rho$ and $t_{i-1}$ that will make $A_{2}^{(i)}$ small. Finally, $A_{3}$ will lead to the non-trivial contribution in (3.28).

Estimates for $A_{1}^{(i)}$. We have

$$
\begin{aligned}
& \frac{d}{d \tau}\left\{\partial_{p q}^{2} f\left(z_{\delta}^{(M)}(\tau)\right) W_{p q}^{(M)}\left(\frac{s}{\delta^{2}}, \frac{\rho}{\delta^{2}}, z_{\delta}(\tau), x_{\delta}(\tau)\right)\right\} \\
= & \frac{1}{\delta} \sum_{r=1}^{d} \partial_{p q r}^{3} f\left(z_{\delta}^{(M)}(\tau)\right) W_{p q}^{(M)}\left(\frac{s}{\delta^{2}}, \frac{\rho}{\delta^{2}}, z_{\delta}^{(M)}(\tau), x_{\delta}(\tau)\right) W_{r}^{(M)}\left(\frac{\tau}{\delta^{2}}, z_{\delta}^{(M)}(\tau), x_{\delta}(\tau)\right) \\
& +\frac{1}{\delta} \sum_{r=1}^{d} \partial_{p q}^{2} f\left(z_{\delta}^{(M)}(\tau)\right) \partial_{z_{r}} W_{p q}^{(M)}\left(\frac{s}{\delta^{2}}, \frac{\rho}{\delta^{2}}, z_{\delta}^{(M)}(\tau), x_{\delta}(\tau)\right) W_{r}^{(M)}\left(\frac{\tau}{\delta^{2}}, z_{\delta}^{(M)}(\tau), x_{\delta}(\tau)\right) \\
& +\frac{1}{\delta} \sum_{r=1}^{d} \partial_{p q}^{2} f\left(z_{\delta}^{(M)}(\tau)\right) \partial_{x_{r}} W_{p q}^{(M)}\left(\frac{s}{\delta^{2}}, \frac{\rho}{\delta^{2}}, z_{\delta}^{(M)}(\tau), x_{\delta}(\tau)\right) V_{r}\left(\frac{\tau}{\delta^{2}}, x_{\delta}(\tau)\right) .
\end{aligned}
$$

Denote the three sums appearing on the right hand side by $C_{p q}^{j}, j=1,2,3$ respectively.

Lemma 3.12. There exist constants $C, \epsilon>0$ such that

$$
\sup _{i} \sup _{s \in\left[t_{i}, t_{i+1}\right]}\left|\mathbb{E}\left\{\Psi \int_{t_{i-1}}^{s} d \rho \int_{t_{i-1}}^{\rho} C_{p q}^{j} d \tau, A_{\delta, t_{i-1}}^{c}\right\}\right| \leq C \delta^{3+\epsilon}\|\Psi\|_{L^{2}}\|f\|_{4 M, 3}
$$

for $j=1,2,3, \delta \in(0,1]$. Let

Proof. We conduct the proof for $j=1$. The other cases can be done similarly.

$$
W_{p q r}^{(M)}(s, \rho, \tau, z, x):=W_{r}^{(M)}(\tau, z, x) W_{p q}^{(M)}(s, \rho, z, x) .
$$

It suffices to show that there exist constants $C, \epsilon>0$ such that

$$
\begin{aligned}
& \quad\left|\int_{t_{i-1}}^{s} d \rho \int_{t_{i-1}}^{\rho} d \tau \mathbb{E}\left\{\partial_{p q r}^{3} f\left(z_{\delta}^{(M)}(\tau)\right) \Psi W_{p q r}^{(M)}\left(\frac{s}{\delta^{2}}, \frac{\rho}{\delta^{2}}, \frac{\tau}{\delta^{2}}, z_{\delta}^{(M)}(\tau), x_{\delta}(\tau)\right), A_{\delta, t_{i-1}}^{c}\right\}\right| \\
& \leq C \delta^{3+\epsilon}\|\Psi\|_{L^{2}}\|f\|_{4 M, 3}
\end{aligned}
$$


with the constants independent of $i, s$. Using the definition of the event $A_{\delta, t_{i-1}}$ we can estimate the left hand side of (3.32) by

$$
C\|\Psi\|_{L^{2}}\|f\|_{4 M, 3} \delta^{\gamma} \int_{0}^{s-t_{i-1}} w_{(s-\rho) / \delta^{2}} d \rho
$$

where (cf. $(3.22))$

$$
w_{s}:=\left\{\mathbb{E}\left[\sup _{|z| \leq M,|x| \leq \delta^{-2}}|\bar{W}(s, 0, z, x)|^{6}\right]\right\}^{1 / 6}
$$

for any $s \geq \rho$. From Proposition 3.5, with $m=6$, we conclude that for any $\mu>0$ (to be adjusted later on) we can choose an appropriate $C>0$ so that

$$
w_{(s-\rho) / \delta^{2}} \leq C \delta^{-\mu} \sup _{|z| \leq M}\left\{\mathbb{E}\left|\bar{W}\left(\frac{s-\rho}{\delta^{2}}, 0, z, 0\right)\right|^{2}\right\}^{1 / 2}, \quad \forall \delta \in(0,1] .
$$

Computing the conditional expectation appearing in the right hand side of the expression above it follows that

$$
w_{(s-\rho) / \delta^{2}} \leq C \delta^{-\mu} C M\left\{\int_{0}^{K_{0}} e^{-2 k^{2 \beta}(s-\rho) \delta^{-2}} \frac{d k}{k^{2 \alpha-3}}\right\}^{1 / 2} .
$$

Making the substitution $\rho^{\prime}:=(s-\rho) / \delta^{2}$ we can estimate expression (3.33) by

$$
C\|\Psi\|_{L^{2}}\|f\|_{4 M, 3} \delta^{2+\gamma-\mu} \int_{0}^{s / \delta^{2}} d \rho\left\{\int_{0}^{K_{0}} e^{-2 k^{2 \beta} \rho} \frac{d k}{k^{2 \alpha-3}}\right\}^{1 / 2}
$$

Performing the change of variables $k^{\prime}:=k \rho^{1 /(2 \beta)}$ we obtain that the expression above equals

$$
C\|\Psi\|_{L^{2}}\|f\|_{4 M, 3} \delta^{2+\gamma-\mu} \int_{0}^{s / \delta^{2}} \frac{d \rho}{\rho^{(2-\alpha) /(2 \beta)}}\left\{\int_{0}^{K_{0} \rho^{1 /(2 \beta)}} \frac{e^{-2 k^{2 \beta}} d k}{k^{2 \alpha-3}}\right\}^{1 / 2} .
$$

The integral with respect to $\rho$ is not singular at 0 and has an integrable singularity at $\infty$, due to the assumption that $\alpha+2 \beta<2$. Since $\gamma>1$ we can choose $\mu<\gamma-1$, thus (3.31) holds.

It follows from Lemma 3.12 that

$$
\left|A_{1}^{(i)}\right| \leq C \delta^{1+\gamma+\epsilon}\|\Psi\|_{L^{2}}\|f\|_{4 M, 3},
$$

hence, after summation over $i$ we get the estimate

$$
\left|A_{1}\right| \leq C \delta^{1+\epsilon}(u-t)\|\Psi\|_{L^{2}}\|f\|_{4 M, 3} .
$$


Estimates for $A_{2}$. To deduce that

$$
\left|A_{2}\right| \leq C \delta^{\epsilon}(u-t)\|\Psi\|_{L^{2}}\|f\|_{4 M, 3},
$$

it suffices to show the following.

Lemma 3.13. There exist $C, \epsilon>0$ such that

$$
\begin{aligned}
& \sup _{i} \sup _{s \in\left[t_{i}, t_{i+1}\right]} \mid \mathbb{E}\left\{\int_{t_{i-1}}^{s} \widetilde{W}_{p q}^{(M)}\left(\frac{s}{\delta^{2}}, \frac{\rho}{\delta^{2}}, \frac{t_{i-1}}{\delta^{2}}, z_{\delta}^{(M)}\left(t_{i-1}\right), x_{\delta}\left(t_{i-1}\right)\right) d \rho\right. \\
& \left.\times \partial_{p q}^{2} f\left(z_{\delta}^{(M)}\left(t_{i-1}\right)\right) \Psi, A_{\delta, t_{i-1}}^{c}\right\} \mid \leq C\|\Psi\|_{L^{2}}\|f\|_{4 M, 2} \delta^{2+\epsilon} .
\end{aligned}
$$

Proof. Making the substitution $\rho^{\prime}:=\rho / \delta^{2}$, and dropping the primes, we obtain that the expression under the supremum equals

$$
\delta^{2}\left|\mathbb{E}\left\{\partial_{p q}^{2} f\left(z_{\delta}^{(M)}\left(t_{i-1}\right)\right) \Psi \int_{t_{i-1} / \delta^{2}}^{s / \delta^{2}} \widetilde{W}_{p q}^{(M)}\left(\frac{s}{\delta^{2}}, \rho, \frac{t_{i-1}}{\delta^{2}}, z_{\delta}^{(M)}\left(t_{i-1}\right), x_{\delta}\left(t_{i-1}\right)\right) d \rho, A_{\delta, t_{i-1}}^{c}\right\}\right| .
$$

Using elementary rules of computing the conditional expectation of Gaussians we get

$$
\begin{aligned}
& \widetilde{W}_{p q}(s, \rho, 0, z, 0)=2 \int_{-\infty}^{0} \int_{-\infty}^{0} \exp \left\{-\left|k_{1}\right|^{2 \beta}\left(s-u_{1}\right)-\left|k_{2}\right|^{2 \beta}\left(\rho-u_{2}\right)\right\}\left(\left|k_{1}\right|\left|k_{2}\right|\right)^{\beta} \\
& \times\left(e^{i k_{1} \cdot z}-1\right)\left(e^{i k_{2} \cdot z}-1\right) \widetilde{\mathcal{B}}_{p q}\left(d u_{1}, d k_{1} ; d u_{2}, d k_{2}\right)
\end{aligned}
$$

where

$$
\begin{aligned}
& \widetilde{\mathcal{B}}_{p q}\left(d u_{1}, d k_{1} ; d u_{2}, d k_{2}\right) \\
:= & \widehat{\mathcal{B}}_{p}\left(d u_{1}, d k_{1}\right) \widehat{\mathcal{B}}_{q}\left(d u_{2}, d k_{2}\right)-\hat{R}_{p q}\left(k_{1}\right) \delta\left(u_{1}-u_{2}\right) \delta\left(k_{1}+k_{2}\right) d u_{1} d u_{2} d k_{1} d k_{2} .
\end{aligned}
$$

A simple calculation shows that

$$
\begin{aligned}
& \int_{0}^{s} \widetilde{W}_{p q}(s, \rho, 0, z, 0) d \rho \\
= & 2 \int_{-\infty}^{0} \int_{-\infty}^{0} \iint \exp \left\{-\left|k_{1}\right|^{2 \beta}\left(s-u_{1}\right)\right\}\left(\frac{\left|k_{1}\right|}{\left|k_{2}\right|}\right)^{\beta} \\
& \times\left[\exp \left\{\left|k_{2}\right|^{2 \beta} u_{2}\right\}-\exp \left\{-\left|k_{2}\right|^{2 \beta}\left(s-u_{2}\right)\right\}\right] \\
& \quad \times\left(e^{i k_{1} \cdot z}-1\right)\left(e^{i k_{2} \cdot z}-1\right) \widetilde{\mathcal{B}}_{p q}\left(d u_{1}, d k_{1} ; d u_{2}, d k_{2}\right) \\
= & 2 Z_{1}(s, z) Z_{2}(s, z)-2 \bar{Z}(s, z),
\end{aligned}
$$

where

$$
\begin{gathered}
Z_{1}(s, z)=\int_{-\infty}^{0} \int \exp \left\{-\left|k_{1}\right|^{2 \beta}\left(s-u_{1}\right)\right\}\left|k_{1}\right|^{\beta}\left(e^{i k_{1} \cdot z}-1\right) \widehat{\mathcal{B}}_{p}\left(d u_{1}, d k_{1}\right), \\
Z_{2}(s, z)=\int_{-\infty}^{0} \int\left[1-\exp \left\{-\left|k_{2}\right|^{2 \beta} s\right\}\right] \frac{e^{\left|k_{2}\right|^{2 \beta} u_{2}}\left(e^{i k_{2} \cdot z}-1\right)}{\left|k_{2}\right|^{\beta}} \widehat{\mathcal{B}}_{q}\left(d u_{2}, d k_{2}\right),
\end{gathered}
$$


and

$$
\bar{Z}(s, z):=\int \frac{1-e^{-|k|^{2 \beta} s}}{2|k|^{\beta}} e^{-|k|^{2 \beta} s}\left|e^{i k \cdot z}-1\right|^{2} \hat{R}_{p q}(k) d k .
$$

It suffices only to prove that there exist $C_{1}, \epsilon_{1}>0$ such that

$$
\begin{aligned}
& \sup _{s \in\left[\delta^{\gamma-2}, 2 \delta^{\gamma-2}\right]} \sup _{|z| \leq M} \mathbb{E} Z_{1}^{2}(s, z) \leq C_{1} \delta^{\epsilon_{1}}, \\
& \sup _{s \in\left[\delta^{\gamma-2}, 2 \delta^{\gamma-2}\right]} \sup _{|z| \leq M}|\bar{Z}(s, z)| \leq C_{1} \delta^{\epsilon_{1}},
\end{aligned}
$$

and

$$
\sup _{s \in\left[\delta^{\gamma-2}, 2 \delta^{\gamma-2}\right]} \sup _{|z| \leq M} \mathbb{E} Z_{2}^{2}(s, z) \leq C_{1} .
$$

Using (1.5), we conclude that

$$
|\bar{Z}(s, z)| \leq C M^{2} \int_{0}^{K_{0}}\left(1-e^{-k^{2 \beta} \delta^{\gamma-2}}\right) e^{-k^{2 \beta} \delta^{\gamma-2}} \frac{d k}{k^{2 \alpha+2 \beta-3}}
$$

for some constant $C>0$. Performing the change of variables $k^{\prime}:=k \delta^{(\gamma-2) /(2 \beta)}$, we obtain

$$
|\bar{Z}(s, z)| \leq C M^{2} \delta^{(2-\gamma)(2-\alpha-\beta) / \beta} \int_{0}^{+\infty}\left(1-e^{-k^{2 \beta}}\right) e^{-k^{2 \beta}} \frac{d k}{k^{2 \alpha+2 \beta-3}} .
$$

On the other hand, we have

$$
\mathbb{E} Z_{1}^{2}(s, z) \leq C M^{2} \int_{0}^{K_{0}} \frac{e^{-k_{1}^{2 \beta} \delta^{\gamma-2}}}{k_{1}^{2 \alpha-3}} d k_{1} \leq C \delta^{\epsilon_{1}}
$$

for some $\epsilon_{1}>0$, since $\alpha<1$. Analogous computations for $Z_{2}(s, z)$ yield

$$
\mathbb{E} Z_{2}^{2}(s, z) \leq C M^{2} \int_{0}^{K_{0}} \frac{\left[1-e^{-k_{2}^{2 \beta} \delta^{\gamma-2}}\right]^{2}}{k_{2}^{2 \alpha+4 \beta-3}} d k_{2} \leq C .
$$

This finishes the proof of Lemma 3.13.

The limit of $A_{3}$. From the definition of $A_{3}$ we get directly that

$$
\begin{aligned}
& \quad\left|A_{3}-\sum_{i} \sum_{p, q=1}^{d} \int_{t_{i}}^{t_{i+1}} \mathbb{E}\left\{\partial_{p q}^{2} f\left(z_{\delta}^{(M)}\left(t_{i-1}\right)\right) c_{p q}^{(M)}\left(z_{\delta}^{(M)}\left(t_{i-1}\right)\right) \Psi, A_{\delta, t_{i-1}}^{c}\right\} d s\right| \\
& \leq C(u-t)\|f\|_{4 M, 2}\|\Psi\|_{2} \sup _{|z| \leq M} \int_{\delta^{\gamma-2}}^{+\infty} d \rho \int_{\mathbb{R}^{d}} e^{-|k|^{2 \beta} \rho} \operatorname{tr} \hat{R}(k)[1-\cos (k \cdot z)]^{2} d k \\
& \leq C M^{2}(u-t)\|f\|_{4 M, 2}\|\Psi\|_{2} \int_{0}^{K_{0}} \frac{e^{-k^{2 \beta} \delta^{\gamma-2}} d k}{k^{2 \alpha+2 \beta-3}} \\
& \leq C \delta^{(2-\gamma)(2-\alpha-\beta) / \beta}\|f\|_{4 M, 2}\|\Psi\|_{2}(u-t) \int_{0}^{+\infty} \frac{e^{-k^{2 \beta}} d k}{k^{2 \alpha+2 \beta-3}} \leq C \delta^{\epsilon}\|f\|_{4 M, 2}\|\Psi\|_{2}(u-t)
\end{aligned}
$$

for some $\epsilon>0$, provided that $\alpha+\beta<2$. This completes the proof of Proposition 3.10. 
Estimates of $J_{22}$ and $J_{23}$ : sketch of the proof of Proposition 3.11. The estimates of $J_{22}, J_{23}$ go precisely along the lines of the estimates for $J_{21}$. The term $W_{p q}^{(M)}(s, \rho, z, x)$ appearing there should be replaced by

$$
U_{p q}^{(M)}(s, \rho, z, x)=\partial_{z_{q}} W_{p}^{(M)}(s, z, x) W_{q}^{(M)}(\rho, z, x),
$$

or by

$$
R_{p q}^{(M)}(s, \rho, z, x)=\partial_{x_{q}} W_{p}^{(M)}(s, z, x) W_{q}^{(M)}(\rho, z, x),
$$

in the case of $J_{22}$ or $J_{23}$, respectively. The estimates are somewhat easier to come by this time because the differentiation operators correspond to multiplication of the spectrum by the $k$ variable, which lowers the degree of singularity of the denominator. The resulting bounds therefore improve.

The divergence free condition on the field $V(t, x)$ implies

$$
\sum_{q=1}^{d} \mathbb{E} R_{p q}^{(M)}(s, \rho, z, x)=0 .
$$

Note also that

$$
\begin{aligned}
& \sum_{q=1}^{d} \mathbb{E} U_{p q}^{(M)}(s, \rho, z, x) \\
= & \sum_{q=1}^{d}\left[\phi_{M}(z) \partial_{z_{q}} \phi_{M}(z) \bar{W}_{p q}(s-\rho, z)+\phi_{M}^{2}(z) \partial_{z_{q}} \bar{W}_{p q}(s-\rho, z)\right] \\
= & \frac{1}{2} \sum_{q=1}^{d} \partial_{z_{q}} \phi_{M}^{2}(z) \bar{W}_{p q}(s-\rho, z) .
\end{aligned}
$$

The last equality follows from the fact that due to the divergence free condition for the field we have

$$
\sum_{q=1}^{d} \partial_{z_{q}} \bar{W}_{p q}(s, z)=0 .
$$

Using the above facts we conclude that (3.29) and (3.30) hold.

Acknowledgment. This work was supported by NSF grants DMS-0908507 and DMS-0908011. LR was also supported by an NSSEFF Fellowship, and TK by Polish Ministry of Science and Higher Education grant NN 201419139.

\section{REFERENCES}

[1] R. Adler, An Introduction to Continuity, Extrema, and Related Topics for General Gaussian Processes, Institute of Mathematical Statistics Lecture Notes-Monograph Series, 12. Institute of Mathematical Statistics, 1990.

[2] G. Bal, T. Komorowski and L. Ryzhik, Asymptotics of the solutions of the random Schrödinger equation, Archiv. Rat. Mech. Appl., 200, 613-664, 2011.

[3] P. Billingsley, Convergence of Probability Measures, John Wiley \& Sons, Inc., New YorkLondon-Sydney, 253, 1968. 
[4] A. Fannjiang and T. Komorowski, Diffusion approximation for particle convection in Markovian flows, Bull. Pol. Acad. Sci., 48, 253-275, 2000.

[5] A. Fannjiang and T. Komorowski, Fractional Brownian motions in a limit of turbulent transport, Ann. Appl. Probab., 10, 1100-1120, 2000.

[6] J. Garnier and K. Solna, Effective fractional acoustic wave equations in random multiscale media, J. Acoust. Soc. Am., 127, 67-72, 2010.

[7] C. Gomez, Radiative Transport Limit for the Random Schrödinger Equation with Long-Range Correlations, preprint available at http://arxiv.org/pdf/1110.3301v1, 2011.

[8] David Gilbarg and Neil S. Trudinger, Elliptic Partial Differential Equations of Second Order, Springer-Verlag, Berlin, 2001.

[9] H. Kesten and G.C. Papanicolaou, A limit theorem for turbulent diffusion, Commun. Math. Phys., 65, 97-128, 1979.

[10] T. Komorowski and L. Ryzhik, Passive tracer in a slowly decorrelating random flow with a large mean, Nonlin., 20, 1215-1239, 2007.

[11] T. Komorowski and L. Ryzhik, On asymptotics of a tracer advected in a locally self-similar, correlated flow, Asymp. Anal., 53, 159-187, 2007.

[12] A.J. Majda and P.R. Kramer, Simplified models for turbulent diffusion: Theory, numerical modelling and physical phenomena, Phys. Rep., 314, 237-574, 1999.

[13] R. Marty and K. Solna, Acoustic waves in long range random media, SIAM J. Applied Math., 69, 1065-1083, 2009.

[14] Y.A. Rozanov, Stationary Random Processes, Translated from the Russian by A. Feinstein Holden-Day, Inc., San Francisco, Calif.-London-Amsterdam, 211, 1967.

[15] D.W. Stroock and S.R. Srinivasa Varadhan, Multidimensional Diffusion Processes, reprint of the 1997 edition, Classics in Mathematics, Springer-Verlag, Berlin, 2006. 